\title{
Coagulopathy in newly diagnosed pediatric patients with malignancies
}

\author{
Hanslik G*, Schlegel PG and Wiegering V \\ Children's Hospital of the University of Würzburg, Josef-Schneider-Straße 2, 97080 Würzburg, Germany
}

Children with leukemia or other malignant diseases have an increased risk of thrombosis and bleeding complications at diagnosis. Well known are bleeding complications especially in patients with acute myeloid leukemia with the subtype M3 due to hyperfibrinolysis and disseminated intravascular coagulation. However, there is a number of different factors influencing the coagulation in other malignant diseases. These coagulation protein abnormalities are a common complication in patients with malignant diseases, which may rise the cancer associated mortality and morbidity. Abnormalities are mostly detected in the clotting system [1].

It has to be emphasized that bleeding and thrombosis in these cases are caused by multiple factors. In addition to thrombocytopenia and coagulation abnormalities due to the underlying malignant disease other primary (hereditary) and secondary risk factors for bleeding (treatment induced thrombocytopenia and decreased platelet function) and for thrombosis (using a central vascular catheter, pro-coagulatory cytostatic drugs, immobility, frequent infections and higher risk for sepsis) are existing.

A few pediatric studies on this subject are hinting that the coagulopathy may occur as a paraneoplastic phenomenon [2]. Recently we published an article, showing that 8 out of 9 pediatric patients with newly diagnosed malignancies and Factor XIII deficiency recovered the factor deficiency after treatment underlining this thesis [3].

To study this topic further we acquired retrospectively data of 298 pediatric patients, who were newly diagnosed with malignant diseases at the Children's Hospital of the University of Würzburg in the period between 2012 and 2016. Various laboratory values were measured via standardized test and evaluated by a collaborative ring trial.

$2.3 \%$ of our patients developed a thrombosis during treatment initiation. This result is consistent for example with the result of the AIEOP-BFM ALL Register, which presented in $2.4 \%$ of the children a venous thromboembolic event [4]. $1.3 \%$ of the evaluated children suffered from a significant bleeding, also consistent to published studies [5].

Over 30\% (90 out of 298) of these pediatric patients showed abnormalities in at least one clotting parameter. Out of all entities patients with leukemia (77.2\%) had the highest risk to develop a coagulopathy, followed by patients with lymphoma (39.1\%). Protein C, Protein S and Factor XIII have been the most noteworthy parameter, which showed pathological values at a high rate (Protein C 36.9\%, Protein S 30.4\% and Factor XIII 26.0\%; Table 1). Supporting the thesis that risk factors for thrombophilia and the incidence for individual clotting factors deficiencies, especially factor XIII deficiency, is highly underestimated [3].

One year after treatment initiation according to the German standard protocols, we reassessed out of the 90 patients with a coagulopathy at diagnosis 70 patients. In 20 patients the coagulation parameter was not tested, so these were not included in further analysis. After treatment a coagulopathy could not be detected in $70 \%$ (49 out of 70) of our evaluated group. 12 of 70 patients (17.1\%) showed a partial remission in at least one factor. Only in 9 of 70 patients $(12.8 \%)$ the coagulopathy persisted. Out of these 9 patients 3 were diagnosed with a hereditary coagulation disorder. A newly diagnosed coagulopathy did not occur.

21 out of 24 patients $(87.5 \%)$, which presented initially a Factor XIII deficiency, showed after treatment initiation normal values for Factor XIII. This result is highly significantly $(\mathrm{p}<0.01)$. Other pathological values also decline after treatment, but showed no significance, most likely due to the small number of our follow-up group.

This is the first analysis of a big cohort of pediatric patients examine coagulopathies in malignancies. Nearly a third of our patients suffered from a coagulation disorder at diagnosis, but more than $70 \%$ recovered from this disorder after treatment and most of the patients did not show clinical signs or complications due to the coagulopathy. However, preventive procedures were induced in patients with known coagulopathies. The results underline a possible paraneoplastic mechanism, which could cause consequently the laboratory coagulopathy and a clinical venous thromboembolic or bleeding event.

We propose that oncological treatment protocol should include a screening for coagulopathies at diagnosis to assess the individual risk for bleeding and thrombosis, since coagulopathies seem to be paraneoplastic phenomena and could not be obtained e.g. by medical history.

Especially the entities, which showed a higher risk for clotting disorder, such as leukemia and lymphoma should be screened. Furthermore, we would like to encourage our colleagues to establish a registry for coagulopathies to gather robust data and to develop accurately and individual treatment strategies to avoid severe

${ }^{\star}$ Correspondence to: Gregor Hanslik, Children's Hospital of the University of Würzburg, Josef-Schneider-Straße 2, 97080 Würzburg, Germany, Tel: +49 931 201 27728; Fax: +49 931201 27730; E-mail: gregor.hanslik@gmail.com

Received: November 28, 2018; Accepted: December 04, 2018; Published: December 07, 2018 
Table 1. Prevalence of coagulopathy at diagnosis and after treatment grouped in different malignant entities

\begin{tabular}{|c|c|c|c|c|c|c|c|}
\hline & \multicolumn{3}{|c|}{ Coagolupathy at diagnosis } & \multicolumn{4}{|c|}{ Coagolupathy persistent after treatment } \\
\hline & no & yes & Total & no & yes & partial & Total \\
\hline Leukemia & $13(22.8 \%)$ & $44(77.2 \%)$ & $57(100 \%)$ & $22(61.1 \%)$ & $6(16.7 \%)$ & $8(22.2 \%)$ & $36(100 \%)$ \\
\hline Lymphoma & $14(60.9 \%)$ & $9(39.1 \%)$ & $23(100 \%)$ & $7(100 \%)$ & $0(0 \%)$ & $0(0 \%)$ & $7(100 \%)$ \\
\hline Solid tumors & $70(72.9 \%)$ & $26(27.1 \%)$ & $96(100 \%)$ & $13(76.5 \%)$ & $2(11.8 \%)$ & $2(11.8 \%)$ & $17(100 \%$ \\
\hline CNS tumor & $87(91.6 \%)$ & $8(8.4 \%)$ & $95(100 \%)$ & $6(75 \%)$ & $1(12.5 \%)$ & $1(12.5 \%)$ & $8(100 \%)$ \\
\hline Others & $24(88.9 \%)$ & $3(11.1 \%)$ & $27(100 \%)$ & $1(50 \%)$ & $0(0 \%)$ & $1(50 \%)$ & $2(100 \%)$ \\
\hline Total & $208(69.8 \%)$ & $90(30.2 \%)$ & $298(100 \%)$ & $49(70 \%)$ & $9(12.8 \%)$ & $12(17.1 \%)$ & $70(100 \%)$ \\
\hline
\end{tabular}

coagulation complications and reduce the mortality in pediatric patients with a malignant disease further.

\section{Funding information/competing interest}

All authors listed have contributed sufficiently to the project to be included as authors, and all those who are qualified to be authors are listed in the author byline. To the best of our knowledge, no conflict of interest, financial or other, exists.

\section{Referenes}

1. Bick RL (1992) Coagulation abnormalities in malignancy: a review. Semin Thromb Hemost 18: 353-372.
2. Weltermann A, Pabinger I, Geissler K, Jäger U, Gisslinger H, et al. (1998) Hypofibrinogenemia in non-M3 acute myeloid leukemia. Incidence, clinical and laboratory characteristics and prognosis. Leukemia 12:1182-1186.

3. Wiegering V, Andres O, Schlegel PG, Deinlein F, Eyrich M, et al. (2013) Hyperfibrinolysis and acquired factor XIII deficiency in newly diagnosed pediatric malignancies. Haematologica 98: e90-e91. [Crossref]

4. Santoro N, Colombini A, Silvestri D, Grassi M, Giordano P, et al. (2013) Screening for coagulopathy and identification of children with acute lymphoblastic leukemia at a higher risk of symptomatic venous thrombosis: an AIEOP experience. $J$ Pediatr Hematol Oncol 35: 348-355.

5. Sutor AH, Mall V, Thomas KB (1999) Bleeding and thrombosis in children with acute lymphoblastic leukaemia, treated according to the ALL-BFM-90 protocol. Klin Padiatr 211: 201-204.

Copyright: $@ 2018$ Hanslik G. This is an open-access article distributed under the terms of the Creative Commons Attribution License, which permits unrestricted use, distribution, and reproduction in any medium, provided the original author and source are credited. 